\title{
SPECT myocardial perfusion imaging identifies myocardial ischemia in patients with a history of COVID-19 without coronary artery disease
}

\author{
Murat Çap ${ }^{1}(0)$ Önder Bilge ${ }^{1} \cdot$ Cihan Gündoğan $^{2} \cdot$ İsmail Tatıı ${ }^{1}$ Cansu Öztürk ${ }^{1} \cdot$ Ercan Taştan $^{1} \cdot$ Ferat Kepenek $^{2}$.

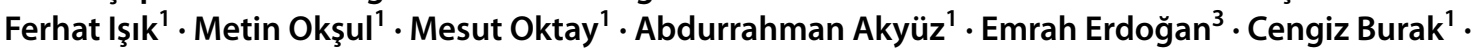 \\ Muhammed Süleymanoğlu ${ }^{1} \cdot$ Ali Karagöz ${ }^{4} \cdot$ İbrahim Halil Tanboğa ${ }^{5}$
}

Received: 16 October 2021 / Accepted: 16 November 2021 / Published online: 22 November 2021

(c) The Author(s), under exclusive licence to Springer Nature B.V. 2021

\begin{abstract}
We aimed to examine the effect of a history of COVID-19 on myocardial ischemia in single-photon emission computed tomography (SPECT) myocardial perfusion imaging (MPI) in patients who presented with shortness of breath and/or chest pain after recovery. For this single-center retrospective study, patients who presented at cardiology outpatient clinics and had SPECT-MPI were screened. A total of 1888 patients were included in the study, 340 of whom had a history of COVID-19. 64 patients with $>50 \%$ stenosis on coronary angiography were excluded from the study. The primary outcome of the study was abnormal MPI. In the study population, the median age was 56 (49-64 IQR) years, and $1127(65 \%)$ of the patients were female. Abnormal MPI was detected in 77 patients (23\%) in the COVID-19 group and in 244 patients (16\%) in the nonCOVID-19 group. After adjustment was performed for clinical predictors using Bayesian logistic regression, an important association was found between the presence of a confirmed prior COVID-19 infection and abnormal MPI (posterior median odds ratio, 1.70 [95\% CrI, 1.20-2.40], risk difference, 9.6\% [95\% CrI, 1.8\%, 19.7\%]). In SPECT-MPI, ischemia rates were observed to be higher in COVID-19 group and it was found that a confirmed prior COVID-19 might predict of abnormal MPI.
\end{abstract}

Keywords COVID-19 $\cdot$ Myocardial ischemia $\cdot$ SPECT $\cdot$ Myocardial perfusion imaging

\section{Introduction}

Although nearly two years have passed since the beginning of the Coronavirus disease 2019 (COVID-19) pandemic, and vaccines are widely used around the world, COVID-19

Murat Çap

murat00418@hotmail.com

1 Department of Cardiology, University of Health Sciences Diyarbakır Gazi Yaşargil Education and Research Hospital, Diyarbakır, Turkey

2 Department of Nuclear Medicine, University of Health Sciences Diyarbakır Gazi Yaşargil Education and Research Hospital, Diyarbakır, Turkey

3 Department of Cardiology, Yüzüncü Y1l University Faculty of Medicine, Van, Turkey

4 Department of Cardiology, University of Health Sciences Kartal Koşuyolu Yüksek İhtisas Education and Research Hospital İstanbul, Istanbul, Turkey

5 Department of Biostatistics and Cardiology, Nişantaş1 University Faculty of Medicine, Istanbul, Turkey continues to be an important cause of mortality and morbidity. Respiratory failure is the primary cause of death in COVID-19 patients, but cardiovascular complications such as acute myocardial infarction (AMI), myocardial injury, fulminant myocarditis, arrhythmias, and thrombotic events may contribute to these patients' morbidity and mortality [1]. In addition to such complications occurring in symptomatic patients, findings of cardiac involvement have also been observed in asymptomatic or mildly symptomatic cases [2,3]. Symptoms such as chest pain, shortness of breath, and fatigue may occur in patients after recovery, along with symptoms occurring during the active period of the disease. Post-acute COVID-19 syndrome is defined as persistent symptoms and/or delayed or long-term complications exceeding four weeks from the onset of symptoms [4]. In a study by Carfi et al., $43 \%$ of patients had shortness of breath and $21 \%$ had chest pain at an average follow-up of 60 days from hospital discharge [5]. Chest pain was reported in 5\% of patients in a Chinese post-acute COVID-19 study with a follow-up of 6 months [6]. In a study conducted by Agustin et al., in which COVID-19 patients who were not 
hospitalized were evaluated, it was determined that $8.7 \%$ of the patients had shortness of breath at their four-month follow-up [7].

It is understood that COVID-19 may cause coronary microvascular dysfunction (CMD) for reasons including an imbalance in the renin-angiotensin-aldosterone system (RAAS), endothelial damage, and microthrombi [8]. In a study performed with stress cardiac magnetic resonance (CMR) imaging in patients with exertional dyspnea and fatigue symptoms after recovery from COVID-19, a decrease in coronary myocardial perfusion reserve was observed [9]. Again, in a 42-year-old patient, coronary computed tomography angiography (CCTA) performed due to persistent exertional angina after COVID-19 infection was observed to be normal. Stress CMR examination of the same patient revealed subendocardial ischemia, which was thought to be due to CMD that disappeared during rest [10]. There is insufficient data on the effect of COVID-19 on myocardial ischemia in myocardial perfusion scintigraphy. We observed that patients who applied to cardiology outpatient clinics with chest pain and shortness of breath after recovery from COVID-19 were numerous, and single-photon emission computed tomography (SPECT) myocardial perfusion imaging (MPI) was undertaken to investigate ischemia in these patients. In this study, we aimed to examine the relation of a history of COVID-19 with ischemia using SPECT-MPI in patients who presented at cardiology outpatient clinics with chest pain and/or shortness of breath after recovery.

\section{Methods}

For this single-center retrospective study, patients who applied to the cardiology outpatient clinics of our hospital between January 1 and June 30, 2021, with chest pain and/ or shortness of breath and who had undergone SPECT-MPI were screened. Patients with a history of coronary revascularization (surgery or percutaneous intervention), AMI, prior critical coronary stenosis with/without revascularization, known heart failure, severe valvular disease such as aortic stenosis, and hypertrophic cardiomyopathy, as well as patients who could not perform optimal MPI, were excluded from the study. After these exclusion criteria were applied, patients who had no known history of cardiac disease and had scarring or both scarring and ischemia $(n=21)$ in SPECT-MPI (newly developed due to MI or myocarditis, etc.) were excluded from the study because the purpose of the study was examining ischemia in SPECT-MPI. Approximately 2920 patients were screened, and after all exclusion criteria were applied, 1888 patients, 340 of whom had a history of COVID-19 infection, were included in the study. A confirmed prior COVID-19 infection was defined as a history of real-time reverse transcriptase polymerase chain reaction test positivity for COVID- 19 .

The demographic, laboratory parameters, comorbidities, angiographic results, and medications of the patients were collected from the electronic medical records of the hospital and the national electronic medical record system. Patients who underwent coronary angiography (CAG) and patients with critical coronary stenosis were identified. Stenosis greater than $50 \%$ in any coronary artery was defined as critical stenosis. Left ventricular ejection fraction (\%) was measured by the biplane Simpson method, Teichholz method, or visual assessment.

This study was approved by the local institutional ethics committee. The study protocol conformed to the Declaration of Helsinki.

\section{Myocardial perfusion imaging}

A two-day stress/rest imaging protocol applying Technetium 99-m methoxy-isobutyronitrile (Tc99m MIBI) was conducted to assess myocardial perfusion. Pharmacological stress was preferred for stress imaging due to the COVID-19 pandemic. We applied adenosine (140 $\mu \mathrm{g} / \mathrm{kg} / \mathrm{min}$ for $6 \mathrm{~min})$ infusion as a radiopharmaceutical agent, as recommended by the European Association of Nuclear Medicine [11]. Imaging commenced 30 to $45 \mathrm{~min}$ after a $600-900 \mathrm{MBq}$ Tc- $99 \mathrm{~m}$ MIBI infusion. A similar dose for resting imaging was given whenever any suspected perfusion defects were detected in the stress images.

\section{Imaging protocol for SPECT}

All images were taken over a $180^{\circ}$ angle orbit from the right anterior oblique, a $45^{\circ}$ angle to the left posterior oblique, and $45^{\circ}$ angle using a dual-head $\gamma$-camera (General Electric Optima NM/CT 640, GE Healthcare, Wauwatosa, WI, USA). The images were provided with a $64 \times 64$ matrix, ultra-high-resolution collimator, and an elliptic orbit with step and shoot acquisition at $3^{\circ}$ interims over $180^{\circ}$ angle, 60 projections, and $9-13 \mathrm{~s}$ per projection, applying a $20 \%$ power window focused on the $140 \mathrm{keV}$ photopeak of Tc-99 m.

The patients were in the supine position during imaging. Image sets acquired by SPECT analysis were reproduced on a dedicated workstation (Xeleris, GE Healthcare, Haifa, Israel) utilizing WBR and Evolution for Cardiac approved company relative risk and noise attenuation parameters, with and without computed tomography (CT) based AC (12 iterations and 10 subsets). A single low-dose chest CT scan (100 keV; $1.0 \mathrm{~mA} ; 0.2-0.3 \mathrm{mS}$ ) was conducted to achieve attenuation maps that could be automatically implemented by the processing software to improve the emission data at the end of each acquisition. The MPI dataset was thoroughly 
rematched with the CT attenuation map to generate the attenuation-edited images.

\section{Imaging interpretation}

The scores that included both the scope and severity of perfusion defects utilizing standard segmentation of 17 myocardial zones were calculated using an automatic software program (e-soft, 2.5, QGS/QPS, Cedars-Sinai Medical Center, Los Angeles, CA, USA) [12]. The sum of scores from the 17 segments of the stress images was represented as the summed stress score (SSS), which described the total abnormal myocardium. An SSS > 3 was accepted as abnormal MPI for ischemia. Total perfusion defect (TPD), indicating a compound of both the severity and scope of the myocardial defect, was also assessed and classified by the presence of $\geq$ $10 \%$ abnormal myocardium [13]. Two experienced readers, blinded to all clinical patient information, interpreted the MPI used in the present study. A filling defect consistent with ischemia in the anterior wall of the SPECT/CT MPI of a patient with a history of COVID-19 and no critical stenosis on the coronary angiogram is shown in Fig. 1.

\section{Statistics}

Continuous variables were presented as mean \pm standard deviation. Categorical variables were presented as counts and percentages. The primary outcome of the study was abnormal MPI. A history of COVID-19 was identified as the main exposure. Age, gender, diabetes mellitus (DM) status, presence of hypertension (HT), smoking status, creatinine levels, hemoglobin levels, low-density lipoprotein cholesterol (LDL) levels, high-density lipoprotein cholesterol (HDL) levels, and C-reactive protein (CRP) levels were chosen as adjustment variables to quantify the relationship between a history of COVID-19 and abnormal MPI. To quantify the association between abnormal MPI and a history of COVID-19, we used Bayesian multivariable logistic regression. The model was fit using a Markov chain Monte Carlo (MCMC) method with four chains, 2000 iterations, and a burn-in of 1000 samples. This was done for each one, drawing iteratively from the posterior distribution and allowing for the calculation of odds ratios (OR) with 95\% credible intervals (CrI). The median posterior odds ratio and 95\% CrI were presented. An OR > 1 indicated that a history of COVID-19 was associated with any increased risk of abnormal MPI. An OR $>1.3$ indicated that a history of COVID-19 was associated with a moderately increased risk of abnormal MPI. We also found the posterior probability of the increased risk of abnormal MPI (Pr [OR > 1]) and the moderately increased risk of abnormal MPI ( $\mathrm{Pr}[\mathrm{OR}>1.3])$ in the presence of a history of COVID-19. Here, we categorized the prior results of Student's t-tests as weakly informative $(\mathrm{df}=7$,

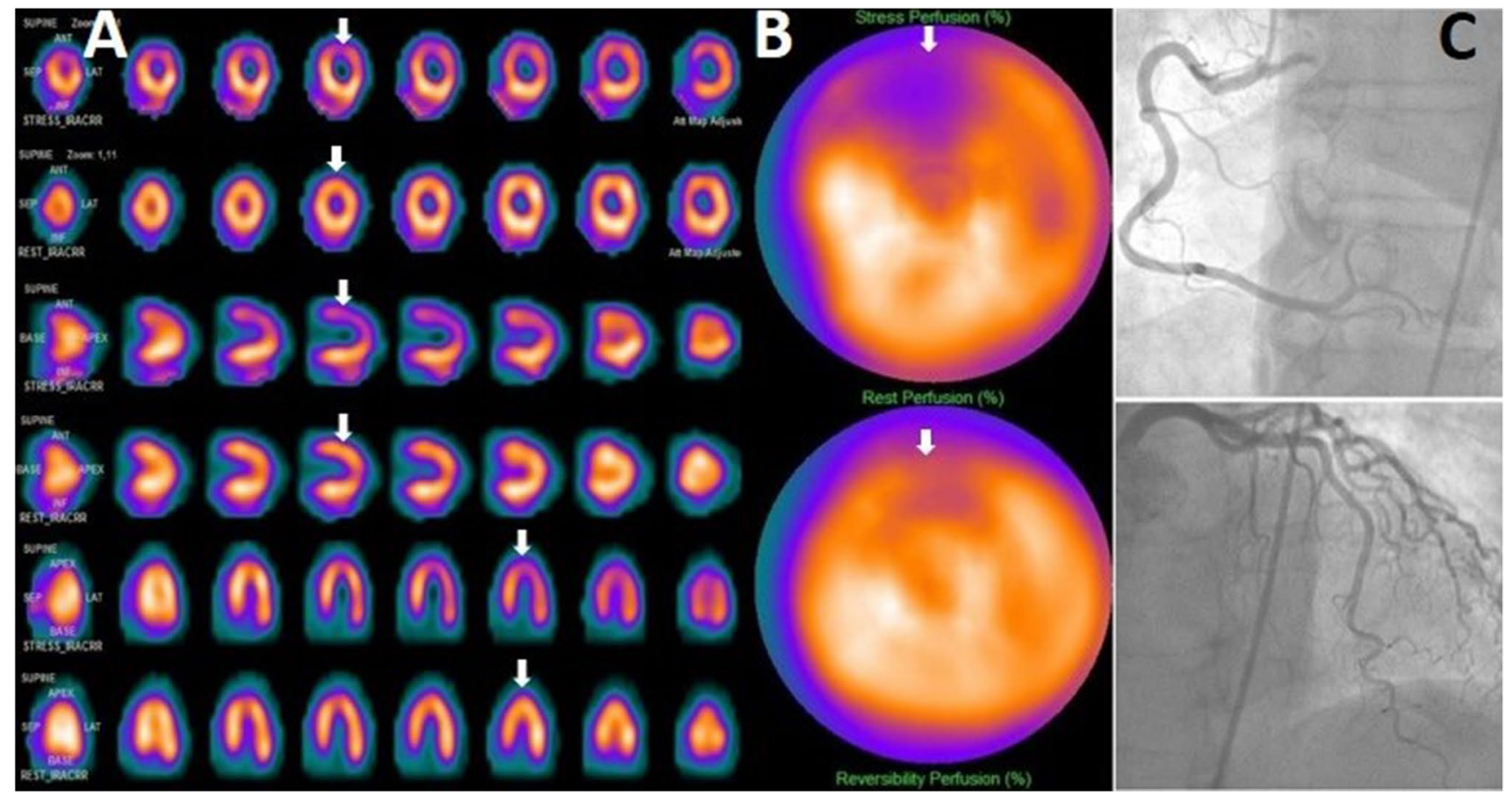

Fig. 1 Stress and rest short-axis, vertical long-axis, and horizontal long-axis images (A) and polar plot images (B) in the MPI of a female patient with a history of COVID-19 show a reversible filling defect (arrows) in the anterior wall consistent with ischemia. Coronary artery stenosis was not observed in the patient's coronary angiography $(\mathbf{C})$ 
$\mathrm{m}=0, \mathrm{~s}=2.5)$, moderately skeptical $(\mathrm{df}=7, \mathrm{~m}=0, \mathrm{~s}=$ $0.25)$, moderately optimistic $(\mathrm{df}=7, \mathrm{~m}=0.25, \mathrm{~s}=0.15)$, and moderately pessimistic $(\mathrm{df}=7, \mathrm{~m}=-0.25, \mathrm{~s}=0.15)$ both for the regression coefficients and the intercepts. In the analysis using different priors, since the data was the same, a heterogeneity to be detected in the meta-analysis will show the effect of the priors on the results. In the meta-analysis, a moderate skeptical prior $[\mathrm{N}(0,0.355)]$, for the overall pooled effect, DuMouchel prior $[(\mathrm{P}(\mathrm{t})=$ $\mathrm{s} 0 /(\mathrm{s} 0+\mathrm{tau}) 2)$, where $\mathrm{S} 0$ is the harmonic mean of the standard errors] for heterogeneity (tau) was used. Finally, we tested the association between time from COVID-19 and MPI and SSS using Bayesian linear regression. Age, gender, DM, HT, smoking, creatinine, hemoglobin, LDL, HDL, and CRP were chosen as adjustment variables. Here, we used weakly informative prior for regression the coefficients $(0,5)$ and intercepts $(0.10)$. The median posterior regression coefficient (Beta) and $95 \% \mathrm{CrI}$ were presented. A Beta $>0$ indicated that the time from COVID-19 to MPI was associated with the SSS. We also presented the posterior probability of Beta $>0$. All statistical analyses were performed using "rstanarm," "bayesmeta," and "ggplot2" packages with R studio version 3.6.3 (R Project, Vienna, Austria).

\section{Results}

A total of 1,888 patients who applied to the cardiology outpatient clinic and requested MPI between January 1 and June 30,2021 , were included in the study. In the study population, the median age was 56 (49-64 IQR) years, and 65\% $(\mathrm{n}=$ $1227)$ of participants were female. There were $340(18 \%)$ patients with a history of COVID-19. All of these patients were symptomatic in the acute period of COVID-19. Thorax computerized tomography was performed in 206 patients, and pneumonia was detected in 127 of these patients. Again, it was determined that 36 patients were hospitalized. The time from COVID-19 to MPI was 150 days (82.5-206.5 IQR). While $26 \%(\mathrm{n}=88)$ of the 340 patients with a history of COVID-19 had abnormal MPI for ischemia findings ( $\mathrm{n}: 46, \mathrm{TPD}>10 \%$ ), CAG was performed in 64 of these patients, and coronary stenosis was detected in $>50 \%$ of 11 patients. While $19 \%(n=297)$ of 1,548 patients without a history of COVID-19 had abnormal MPI for ischemia findings (n:122 TPD > 10\%), 192 of these patients underwent CAG angiography and 53 had $>50 \%$ coronary stenosis (Fig. 2). To evaluate the study's hypothesis (myocardial ischemia in non-obstructive coronaries) more precisely, 64 patients with $>50 \%$ stenosis in CAG, 11 of whom were

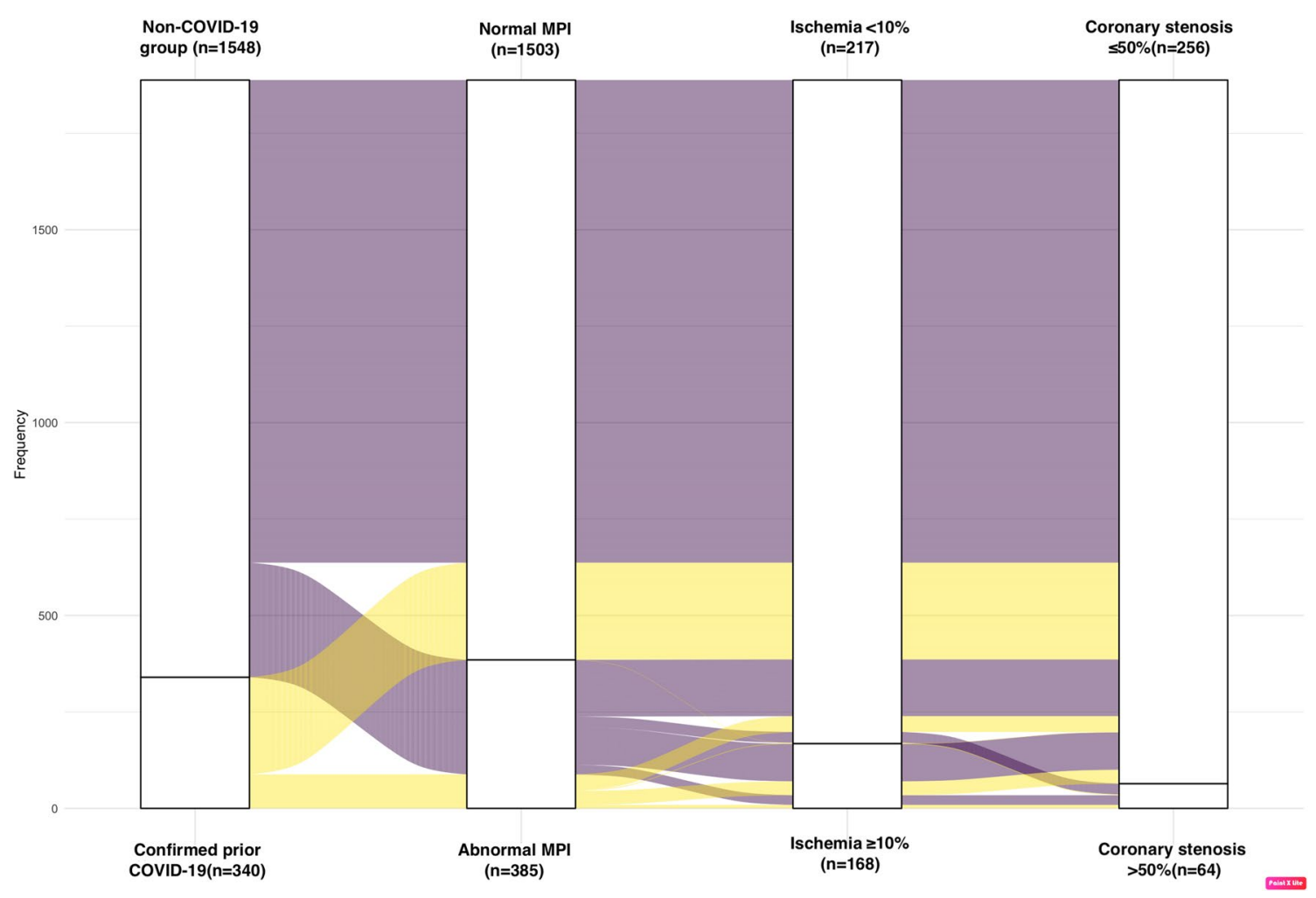

Fig. 2 Stratification of the study population according to the history of COVID-19, abnormal MPI, ischemia burden $>10 \%$, and coronary stenosis $>\% 50$ in coronary angiography in alluvial plot 
COVID-19 and 53 were non-COVID-19, were excluded. The table below summarizes the baseline demographic, clinical, and laboratory characteristics of patients with a history of $\operatorname{COVID}(\mathrm{n}=329)$ and without $(\mathrm{n}=1495$; Table 1$)$.

Abnormal MPI findings were detected in 77 patients (23\%) in the group with a history of COVID-19 and in 244 patients (16\%) in the group without a history of COVID-19. After adjustment for age, gender, DM, HT, smoking, creatinine, hemoglobin, LDL, HDL, and CRP using Bayesian logistic regression, an important association was found between the presence of a history of COVID-19 and abnormal MPI findings (Table 2). When a weakly informative prior was used, the posterior median odds ratio was 1.70 (95\% CrI, 1.20-2.40), and the estimated risk difference was 9.6\% (95\% CrI, 1.8\%, 19.7\%). There was a 99.9\% probability that the OR was greater than 1 (this indicated any increase in the risk of abnormal MPI) and a $93.4 \%$ probability that the OR was greater than 1.3 (this indicated a moderate increase
Table 1 Baseline clinical and laboratory characteristics of patients according to COVID19 history

\begin{tabular}{|c|c|c|c|}
\hline & $\begin{array}{l}\text { Group A: confirmed } \\
\text { prior COVID-19 } \\
\mathrm{n}=329\end{array}$ & $\begin{array}{l}\text { Group B: non- } \\
\text { COVID-19 } \\
n=1495\end{array}$ & $\begin{array}{l}\text { Posterior probability of } \\
\text { difference }^{\mathrm{a}}(\mathrm{A}-\mathrm{B})>0\end{array}$ \\
\hline Sex (female), n (\%) & $220(67 \%)$ & $979(65 \%)$ & 0.679 \\
\hline Age (years) & $55.8 \pm 10.4$ & $56.1 \pm 11.5$ & 0.309 \\
\hline Diabetes mellitus n (\%) & $84(26 \%)$ & $329(22 \%)$ & 0.916 \\
\hline Hypertension n (\%) & $160(49 \%)$ & $756(51 \%)$ & 0.270 \\
\hline Smoking n (\%) & $75(23 \%)$ & $317(21 \%)$ & 0.778 \\
\hline Hyperlipidemia, n (\%) & $81(25.6 \%)$ & $402(27.3 \%)$ & 0.146 \\
\hline Chronic pulmonary disease, $\mathrm{n}(\%)$ & $43(13.1 \%)$ & $156(10.4 \%)$ & 0.918 \\
\hline Cerebrovascular disease, $\mathrm{n}(\%)$ & $6(1.8)$ & $42(2.8)$ & 0.136 \\
\hline \multicolumn{4}{|l|}{ Symptom (main symptom) } \\
\hline Chest pain, n (\%) & $182(55.3 \%)$ & $1238(82.8 \%)$ & \\
\hline Dyspnea, n (\%) & $147(44.7 \%)$ & $257(17.2 \%)$ & $>0.999$ \\
\hline LVEF \% & $60.3 \pm 3.2$ & $59.9 \pm 2.5$ & 0.996 \\
\hline Creatinine & $0.82 \pm 0.43$ & $0.81 \pm 0.54$ & 0.624 \\
\hline White blood cell count, $103 / \mu \mathrm{L}$ & $8.0 \pm 1.96$ & $8.3 \pm 2.3$ & 0.014 \\
\hline Hemoglobin g/dL & $13.8 \pm 1.7$ & $13.7 \pm 1.7$ & 0.901 \\
\hline Platelet, $103 / \mu \mathrm{L}$ & $268 \pm 73$ & $275 \pm 72$ & 0.071 \\
\hline LDL-cholesterol, mg/dL & $116 \pm 35$ & $112 \pm 33$ & 0.949 \\
\hline HDL -cholesterol, mg/dL & $47.5 \pm 12.5$ & $46.5 \pm 11.8$ & 0.889 \\
\hline Triglyceride, $\mathrm{mg} / \mathrm{dL}$ & $191 \pm 114$ & $174 \pm 112$ & 0.986 \\
\hline C-reactive protein, $\mathrm{mg} / \mathrm{dL}$ & $4.6 \pm 4.9$ & $4.34 \pm 5.2$ & 0.786 \\
\hline Summed Stress Score & $1.74 \pm 3.09$ & $1.06 \pm 2.31$ & $>0.999$ \\
\hline Abnormal MPI, n (\%) & $77(23 \%)$ & $244(16 \%)$ & 0.999 \\
\hline Total perfusion defect $>10 \%$ & $37(11 \%)$ & $97(6.0 \%)$ & 0.997 \\
\hline \multicolumn{4}{|l|}{ Medications } \\
\hline ACEI/ARB, n (\%) & $144(43.8)$ & $670(44.9)$ & \\
\hline Beta-blocker, n (\%) & $125(38.1)$ & $580(38.8)$ & \\
\hline Nitrate, n (\%) & $5(1.5)$ & $22(1.5)$ & \\
\hline Ranolazine, n (\%) & $2(0.6)$ & $13(0.9)$ & \\
\hline $\mathrm{CCB}, \mathrm{n}(\%)$ & $86(26.1)$ & $368(24.6)$ & \\
\hline Trimetazidine, n (\%) & $11(3.4)$ & $51(3.4)$ & \\
\hline Lipid lowering agents, n (\%) & $57(17.3)$ & $294(19.7)$ & \\
\hline Antiplatelets, n (\%) & $155(47.1)$ & $555(37.2)$ & \\
\hline Oral antidiabetics, n (\%) & $80(24.5)$ & $318(21.3)$ & \\
\hline Insulin, n (\%) & $29(8.8)$ & $86(5.8)$ & \\
\hline
\end{tabular}

$C C B$ calcium channel blocker; $A C E I / A R B$ angiotensin converting enzyme inhibitor/angiotensin receptor blocker; $L D L$ low-density lipoprotein; $H D L$ high-density lipoprotein; $L V E F \%$ left ventricular ejection fraction

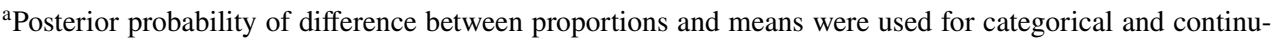
ous variables, respectively (posterior probability shown between 0 and 1) 
Table 2 Evaluation of the relationship between the confirmed prior COVID-19 and abnormal MPI with Bayesian multivariable logistic regression using non-informative prior

\begin{tabular}{lll}
\hline Variables & $\begin{array}{l}\text { Posterior median odds ratio, } \\
(95 \% \text { credible interval })\end{array}$ & $\begin{array}{l}\text { Posterior probability of } \\
\text { abnormal MPI (probability of } \\
\text { OR }>1)\end{array}$ \\
\hline Confirmed prior COVID-19 (yes) & $1.70(1.20-2.40)$ & 0.999 \\
Age (10-unit increase, years) & $0.94(0.82-1.07)$ & 0.162 \\
Sex (female) & $0.80(0.56-1.16)$ & 0.121 \\
Diabetes mellitus (yes) & $1.14(0.81-1.62)$ & 0.781 \\
Hypertension (yes) & $0.86(0.63-1.18)$ & 0.174 \\
Smoking (yes) & $1.47(1.06-2.03)$ & 0.989 \\
Creatinine (1-unit increase, mg/dl) & $0.86(0.56-1.13)$ & 0.162 \\
Hemoglobin (1-unit increase, g/dl) & $1.03(0.93-1.13)$ & 0.722 \\
LDL (10-unit increase, mg/dl) & $0.98(0.94-1.02)$ & 0.180 \\
HDL (5-unit increase, $\mathrm{mg} / \mathrm{dl})$ & $1.00(0.94-1.06)$ & 0.440 \\
CRP (1-unit increase, $\mathrm{mg} / \mathrm{dl})$ & $1.01(0.98-1.04)$ & 0.813 \\
\hline
\end{tabular}

$L D L$ low-density lipoprotein; $H D L$ high-density lipoprotein; $C R P$ C-reactive protein; $L D L$ low density lipoprotein in the risk of abnormal MPI; Fig. 3). Analyses using different priors are shown in Table 3. In summary, for all priors, there was $>90 \%$ probability that the OR was greater than 1 (this indicated any degree of the increasing risk of abnormal MPI). In addition, we evaluated the effect of different priors on posterior probability distribution in a meta-analysis context. Estimated heterogeneity was a low 0.093 (there was only $9.3 \%$ variance among the analysis results when using different priors, which indicated small changes caused by different priors used with Bayesian analysis; Fig. 4). This showed that our Bayesian analysis was robust relative to various priors.

Finally, we used Bayesian multivariable linear regression to test the relationship between time from COVID-19 to the MPI and SSS. Each 30-day increase in time from COVID19 to MPI was associated with an increase in the SSS of 0.04 ( $\beta$ ) units $(95 \% \mathrm{CrI},-0.117,0.200)$. There was a $70 \%$ probability that the $\beta$ was greater than 0 (this indicated any

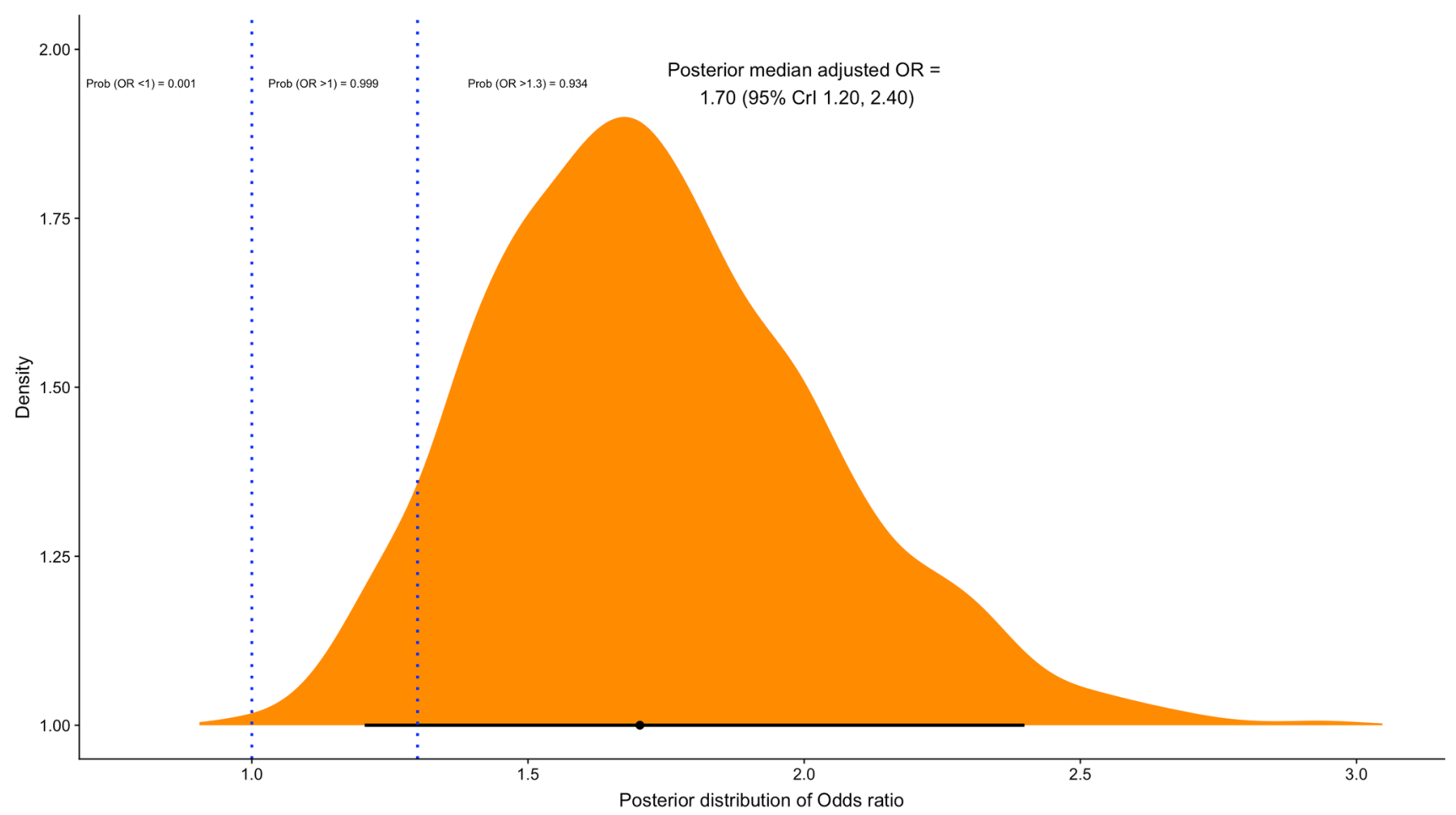

Fig. 3 Posterior probability of odds ratio using weakly informative priors used 
Table 3 Evaluating the relationship between confirmed prior COVID-19 and abnormal MPI using different priors

\begin{tabular}{llll}
\hline Priors & $\begin{array}{l}\text { Posterior median } \\
\text { OR, (95\% CrI) }\end{array}$ & $\begin{array}{l}\text { Posterior probability of } \\
\text { abnormal MPI (probability of } \\
\text { OR }>1)\end{array}$ & $\begin{array}{l}\text { Posterior probability of } \\
\text { abnormal MPI (probability of } \\
\text { OR }>1.3)\end{array}$ \\
\hline Weakly informative & $1.70(1.20-2.40)$ & 0.999 & 0.934 \\
Moderate skeptical & $1.47(1.07-2.02)$ & 0.993 & 0.784 \\
Moderate optimistic & $1.44(1.15-1.86)$ & 0.999 & 0.805 \\
Moderate pessimistic & $1.32(0.93-1.90)$ & 0.937 & 0.525 \\
Frequentist & $1.71(1.21-2.39)^{\mathrm{a}}$ & - & - \\
\hline
\end{tabular}

${ }^{a}$ In frequentist statistics, $95 \%$ confidence interval was used

A

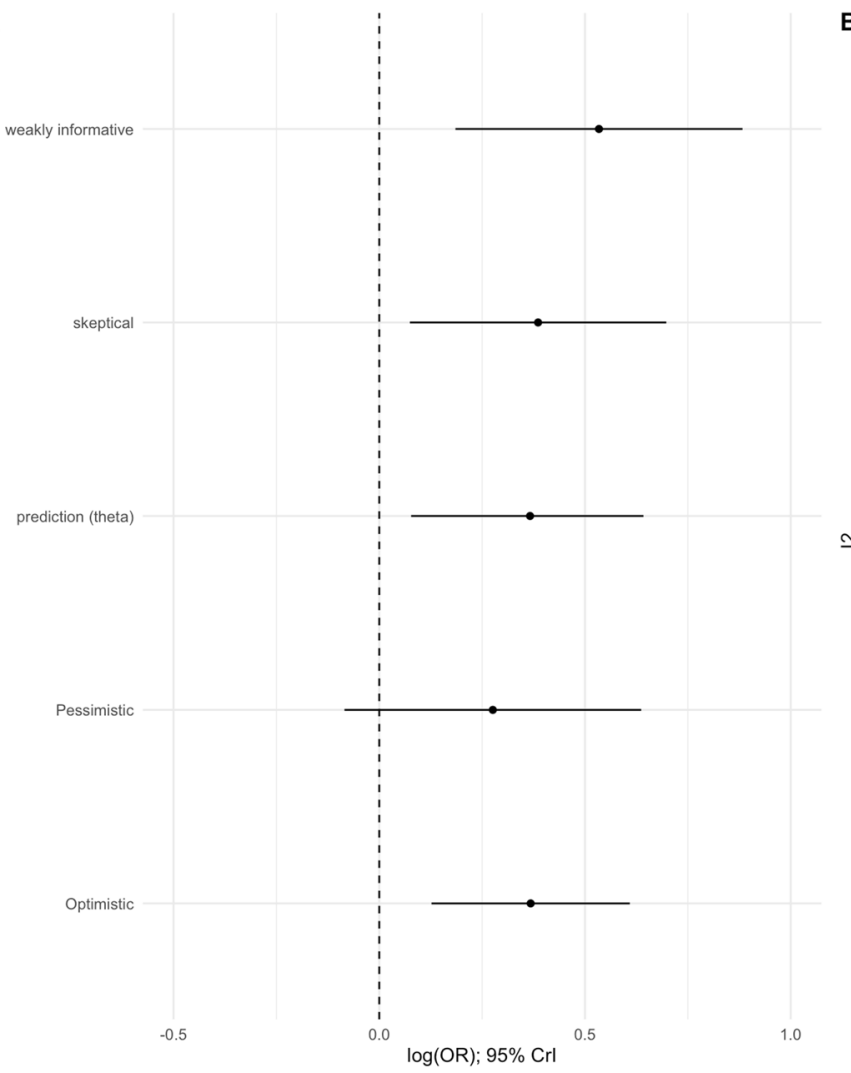

B For median Tau, $9.3 \%$

0.8

0.6

0.2

0.4

0.0 of heterogeneity is explained by priors

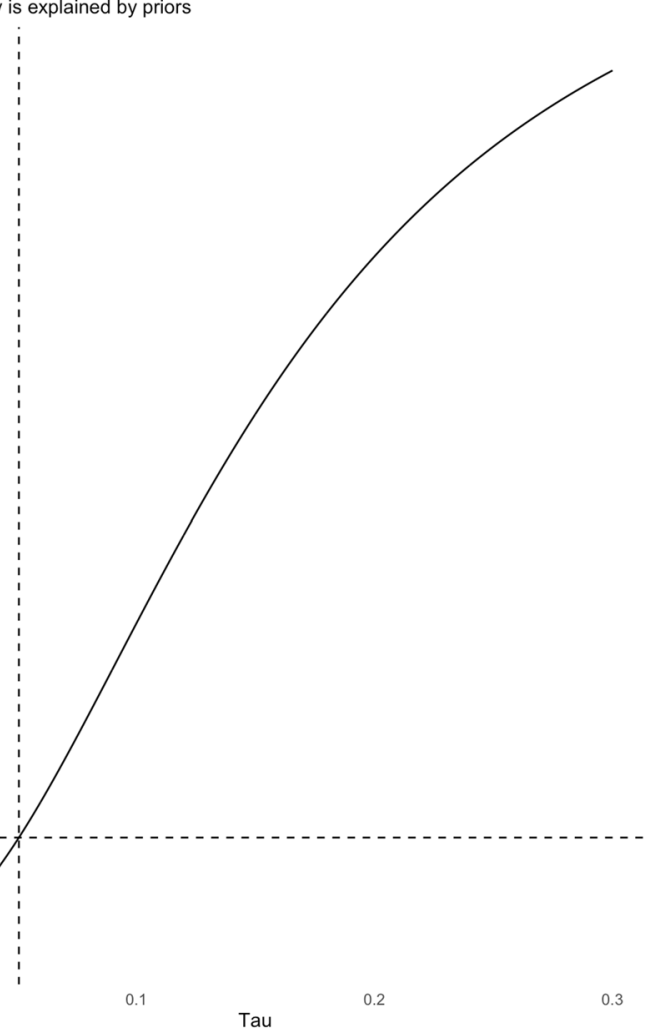

Fig. 4 Forest plot for the results after each prior (A) and resulting heterogeneity $\left(\mathrm{I}^{2}\right)(\mathbf{B}) . \sim 9.3 \%$ of the heterogeneity is explained by the priors. This suggests that our results are relatively robust to when the different priors used

degree of increase in the SSS) and a $24 \%$ probability that the $\beta$ was greater than 0.1 (this indicated a mild increase in the SSS).

\section{Discussion}

This is the first study to evaluate myocardial perfusion scintigraphy in COVID-19 patients who presented with chest pain and/or shortness of breath after recovery. The results of the study showed us that the rates of abnormal MPI for ischemia were higher in the COVID-19 group and that a history of COVID-19 could predict ischemia in MPI (Odds-Ratio was greater than 1.3 with $93.4 \%$ probability). In addition, there was a weak relationship between time from COVID-19 to MPI and SSS.

In COVID-19, which continues its effect all over the world, important cardiovascular pathologies may occur in addition to respiratory tract involvement. During the active period of the disease, AMI, myocarditis, stress cardiomyopathy, myocardial injury, and arrhythmias may occur [1, 14]. Based on current knowledge, proposed mechanisms of cardiac injury are the direct entry of the virus and the 
resultant myocardial damage, systemic inflammation, hypoxia, cytokine storm, and plaque destabilization [14].

In addition to causing cardiac complications during the active period, COVID-19 can also cause symptoms after recovery, and signs of cardiac involvement can be encountered. Studies have shown that symptoms such as shortness of breath, chest pain, and fatigue after recovery from the disease exist [4]. Although more common in hospitalized patients, these complaints may occur during the post-COVID-19 period in those who are not hospitalized and have mild symptoms [5-7]. In a CMR study conducted in COVID-19 patients with symptomatic disease (at home or hospitalized) in which 100 patients were evaluated after recovery, cardiac involvement was found in $78 \%$ of the patients, and myocardial inflammation was detected in $60 \%$ of the CMR taken after a median of 71 days [15]. Again, in a CMR study evaluating 26 athletes who had asymptomatic or mild symptoms of COVID-19, findings consistent with myocarditis were found in four (15\%) of the patients [3]. In a meta-analysis evaluating studies of patients undergoing cardiac evaluation in the post-COVID-19 period, the median time from diagnosis or recovery to cardiac evaluation was 48 days (1-180 days). In these patients, pericardial effusion (15\%) and late gadolinium enhancement (11\%) were detected in CMR, with symptoms like chest pain (25\%) and dyspnea (36\%) in patients evaluated within the first three months. Common changes were detected at approximately three to six months after infection, including left ventricular global longitudinal strain reduction (30\%) and late gadolinium enhancement (10\%) in CMR. Additionally, COVID-19 survivors were at greater risk of developing heart failure, arrhythmias, and myocardial infarction [16].

It is understood that COVID-19 may increase the risk of AMI by causing instability of the atherosclerotic plaque in the epicardial coronary arteries, as well as causing myocardial ischemia due to CMD $[1,8,16]$. In a CMR study conducted with 148 patients after discharge from COVID-19 with myocardial injury, adenosine stress perfusion CMR was performed in 76 of these patients, and inducible ischemia was detected in 20 (26\%) of these patients (seven had an additional infarct area) [17]. In a study by Drakos et al. in patients with shortness of breath and post-covid fatigue syndrome after recovery from COVID-19, it was found that myocardial perfusion reserve with CMR was significantly lower in the COVID-19 group than in the healthy group [9]. In our study, ischemia was observed in the MPI of 77 (23\%) of the patients in the COVID-19 group. The rate of ischemia using SPECT-MPI was observed to be higher in the COVID19 group, and it was determined that a history of COVID-19 could be a predictor of abnormal MPI.

SARS-CoV-2 infects host cells through the transmembrane angiotensin-converting enzyme 2 (ACE2) receptor, which is expressed in many organs and tissues such as the heart, lungs, and kidneys, including vascular endothelial cells $[18,19]$. Emerging evidence has suggested that COVID-19 is associated with RAAS dysregulation, hyperinflammation, and coagulation dysfunction endothelial dysfunction [20-22]. These mechanisms may all cause structural and functional changes in the microvascular circulation, resulting in deterioration in organ perfusion and metabolism and direct organ damage [8, 23]. In the heart, one of the organs connected to microvascular circulation, hemodynamics and metabolic homeostasis are regulated by the coronary microvasculature $[8,24]$. In primary CMD without obstructive coronary artery disease, functional and structural abnormalities in the coronary microvasculature impair the vessels' ability to increase myocardial blood flow and vasodilation function, leading to ischemia and angina if myocardial oxygen demand is increased [25]. SARS-Cov-2 causes the down-regulation of ACE-2, which is involved in the inactivation of angiotensin II; in addition, the hyperactivation of angiotensin II causes vasoconstriction, hypertrophy, fibrosis, and the release of reactive oxygen species [26]. Coronary microvessels can be damaged directly by SARS-CoV-2, hyper inflammation, ARDS-induced hypoxia, and autonomic nerve dysfunction, or indirectly due to damage to perivascular cells. Again, there may be occlusions in coronary microvessels due to atherosclerotic fragments and microthrombi [8]. In the study of Drakos et al., they proposed that decreased myocardial perfusion reserve might be due to coronary microvascular dysfunction. Furthermore, in a brief report, which included a small number of patients, in which CMD was evaluated as invasive, it was determined that ischemia detected in SPECT-MPI without functional and anatomical critical stenosis in the coronary arteries may be due to microvascular dysfunction and can be used as a prognostic factor for severe cardiac events [27]. Although we did not make an invasive evaluation for CMD in our study, increasing ischemia with MPI in the COVID-19 group might be due to CMD in patients without critical stenosis in $\mathrm{CAG}$.

Considering both our clinical practice and the results of previous studies, the number of patients presenting with chest pain and shortness of breath in the post-COVID-19 period is significant [5-7]. The symptoms in patients with ischemia in SPECT-MPI and no critical coronary stenosis on CAG suggest that we should be mindful of COVID-19 for risk assessment. CMD after the COVID-19 period is conceivable when assessing patients with chest pain or dyspnea during the chronic period. In patients with low/ intermediate probability CAD, to exclude significant coronary stenosis, performing CCTA may reduce the need for invasive CAG, especially in patients with low TPD. 


\section{Conclusions}

Ischemia rates were observed to be higher in MPI in COVID-19 patients compared to those in the non-COVID19 group, and it was found that a confirmed prior COVID-19 infection could be a predictor of abnormal MPI. Symptoms such as chest pain and shortness of breath that occur in some patients in the post-COVID-19 period may be caused by CMD in patients with ischemia in MPI and without critical coronary stenosis.

\section{Limitations}

One of the most important limitations of the study is that it has a single-center and retrospective design. Other important limitations of the study include the inability to perform coronary angiography in all patients with ischemia in MPI due to patient preference and the decrease in interventional procedures due to the pandemic. In addition, we could not assess microvascular dysfunction invasively. Another limitation of the study is that it could not be included in the multivariable analysis due to the lack of family history data on coronary artery disease. Given that COVID-19 is determined by PCR positivity, the inability to identify patients who survived the disease asymptomatically or who had COVID-19 but were PCR negative is another limitation.

\section{Funding None.}

\section{Declarations}

Conflict of interest The authors declare that they have no conflict of interest.

\section{References}

1. Azevedo RB, Botelho BG, Hollanda JVG et al (2021) Covid19 and the cardiovascular system: a comprehensive review. J Hum Hypertens 35(1):4-11. https://doi.org/10.1038/ s41371-020-0387-4

2. Gupta S, Mitra A (2021) Challenge of post-COVID era: management of cardiovascular complications in asymptomatic carriers of SARS-CoV-2. Heart Fail Rev. https://doi.org/10.1007/ s10741-021-10076-y

3. Rajpal S, Tong MS, Borchers J et al (2021) Cardiovascular magnetic resonance findings in competitive athletes recovering from COVID-19 infection. JAMA Cardiol 6(1):116-118. https://doi. org/10.1001/jamacardio.2020.4916

4. Nalbandian A, Sehgal K, Gupta A et al (2021) Post-acute COVID19 syndrome. Nat Med 27(4):601-615. https://doi.org/10.1038/ s41591-021-01283-z

5. Carfî A, Bernabei R, Landi F, Gemelli Against COVID-19 PostAcute Care Study Group (2020) Persistent symptoms in patients after acute COVID-19. JAMA 324(6):603-605. https://doi.org/ 10.1001/jama.2020.12603

6. Huang C, Huang L, Wang Y et al (2020) 6-month consequences of COVID-19 in patients discharged from hospital: a cohort study. Lancet 397(10270):220-232. https://doi.org/10.1016/S01406736(20)32656-8

7. Augustin M, Schommers P, Stecher M et al (2021) Post-COVID syndrome in non-hospitalised patients with COVID-19: a longitudinal prospective cohort study. Lancet Reg Health Eur 6:100122. https://doi.org/10.1016/j.lanepe.2021.100122

8. Yin Yin J, Wang S, Liu Y, Chen J, Li D, Xu T (2021) Coronary microvascular dysfunction pathophysiology in COVID-19. Microcirculation 28:e12718

9. Drakos S, Chatzantonis G, Bietenbeck M et al (2021) A cardiovascular magnetic resonance imaging-based pilot study to assess coronary microvascular disease in COVID-19 patients. Sci Rep 11(1):15667. https://doi.org/10.1038/s41598-021-95277-z

10. Vallejo N, Teis A, Mateu L et al (2021) Persistent chest pain after recovery of COVID-19: microvascular disease-related angina? Eur Heart J Case Rep 5(3):105. https://doi.org/10.1093/ehjcr/ ytab105

11. Verberne HJ, AcampaW, Anagnostopoulos C et al (2015) EANM procedural guidelines for radionuclide myocardial perfusion imaging with SPECT and SPECT/CT: 2015 revision. Eur J Nucl Med Mol Imaging 2015:1929-1940

12. Germano G, Kavanagh PB, Waechter P et al (2000) A new algorithm for the quantitation of myocardial perfusion SPECT. I: technical principles and reproducibility. J Nucl Med 41:712-719

13. Nappi C, Gaudieri V, AcampaW et al (2018) Comparison of left ventricular shape by gated SPECT imaging in diabetic and nondiabetic patients with normal myocardial perfusion: a propensity score analysis. J Nucl Cardiol 25:394-403

14. Magadum A, Kishore R (2020) Cardiovascular manifestations of COVID-19 infection. Cells 9(11):2508. https://doi.org/10.3390/ cells9112508

15. Puntmann VO, Carerj ML, Wieters I et al (2020) Outcomes of cardiovascular magnetic resonance imaging in patients recently recovered from coronavirus disease 2019 (COVID-19). JAMA Cardiol 5(11):1265-1273. https://doi.org/10.1001/jamacardio. 2020.3557

16. Ramadan MS, Bertolino L, Zampino R, Monaldi Hospital Cardiovascular Infection Study Group et al (2021) Cardiac sequelae after coronavirus disease 2019 recovery: a systematic review. Clin Microbiol Infect 27:1250-1261

17. Kotecha T, Knight DS, Razvi Y et al (2021) Patterns of myocardial injury in recovered troponin-positive COVID-19 patients assessed by cardiovascular magnetic resonance. Eur Heart $\mathbf{J}$ 42(19):1866-1878. https://doi.org/10.1093/eurheartj/ehab075

18. Varga Z, Flammer AJ, Steiger P et al (2020) Endothelial cell infection and endotheliitis in COVID-19. Lancet 395:1417-1418

19. Hamming I, Timens W, Bulthuis MLC et al (2004) Tissue distribution of ACE2 protein, the functional receptor for SARS coronavirus. A first step in understanding SARS pathogenesis. J Pathol 203:631-637

20. Alifano M, Alifano P, Forgez P et al (2020) Renin-angiotensin system at the heart of COVID-19 pandemic. Biochimie 174:30-33

21. Huang C, Wang Y, Li X et al (2020) Clinical features of patients infected with 2019 novel coronavirus in Wuhan, China. Lancet 395:497-506

22. Castelli R, Gidaro A (2020) Abnormal hemostatic parameters and risk of thromboembolism among patients with COVID-19 infection. J Hematol 9:1-4

23. Serné EH, de Jongh RT, Eringa EC (2007) Microvascular dysfunction: a potential pathophysiological role inthe metabolic syndrome. Hypertension 50:204-211 
24. Lee J, Niederer S, Nordsletten D et al (2009) Coupling contraction, excitation, ventricular and coronary blood flow across scale and physics in the heart. Philos Trans A Math Phys Eng Sci 367:2311-2331

25. Ayub MT, Kalra D (2020) Coronary microvascular dysfunction and the role of noninvasive cardiovascular imaging. Diagnostics 10(9):679. https://doi.org/10.3390/diagnostics10090679

26. Jin Y, Ji W, Yang $\mathrm{H}$ et al (2020) Endothelial activation and dysfunction in COVID-19: from basic mechanisms to potential therapeutic approaches. Signal Transduct Target Ther 5(1):293. https:// doi.org/10.1038/s41392-020-00454-7
27. Djaïleb L, Riou L, Piliero N et al (2018) SPECT myocardial ischemia in the absence of obstructive CAD: contribution of the invasive assessment of microvascular dysfunction. J Nucl Cardiol 25(3):1017-1022. https://doi.org/10.1007/s12350-017-1135-1

Publisher's Note Springer Nature remains neutral with regard to jurisdictional claims in published maps and institutional affiliations. 